\title{
Combination of resveratrol and green tea epigallocatechin gallate induces synergistic apoptosis and inhibits tumor growth in vivo in head and neck cancer models
}

\author{
A.R.M. RUHUL AMIN ${ }^{1,2}$, DONGSHENG WANG ${ }^{1}$, SREENIVAS NANNAPANENI ${ }^{1}$, \\ RAJAN LAMICHHANE ${ }^{3}$, ZHUO GEORGIA CHEN ${ }^{1}$ and DONG M. SHIN ${ }^{1}$
}

\author{
${ }^{1}$ Department of Hematology and Medical Oncology, Winship Cancer Institute of Emory University, \\ Atlanta, GA 30322; ${ }^{2}$ Department of Pharmaceutical Sciences and Research, Marshall University School of Pharmacy, \\ Huntington, WV 25755; ${ }^{3}$ Department of Clinical and Translational Sciences, John C. Edwards School of Medicine, \\ Marshall University, Huntington, WV 25701, USA
}

Received November 18, 2020; Accepted February 9, 2021

DOI: $10.3892 / o r .2021 .8038$

\begin{abstract}
Despite widespread interest in chemoprevention and therapy due to the high margin of safety of dietary natural compounds, clinical intervention with single agents has failed to yield the expected outcomes, mostly due to poor bioavailability and low potency. Combinations of natural agents with synergistic effects are gaining increasing attention. In the present study, in vitro and in vivo antitumor effects of a combination of two natural dietary agents, green tea epigallocatechin gallate (EGCG) and resveratrol were investigated. It was revealed that their combination at low doses (at which single agents induce minimal apoptosis) synergistically increased apoptosis (combination index $<1$ ) in head and neck cancer cell lines. Synergistic apoptosis was also supported by caspase-3 and PARP cleavage. The combination also significantly inhibited growth of xenografted head and neck tumors in nude mice as supported by significant inhibition of tumor volume, tumor weight and Ki67 expression, and increase in TUNEL-positive cells. Mechanistic studies revealed that the combination inhibited AKT-mTOR signaling both in vitro and in vivo. In addition, overexpression of constitutively active AKT protected cells from apoptosis induced by the combination of EGCG and resveratrol. Collectively, the present results for the first time suggest that the combination of EGCG and
\end{abstract}

Correspondence to: Dr A.R.M. Ruhul Amin, Department of Pharmaceutical Sciences and Research, Marshall University School of Pharmacy, 1 John Marshall Drive, Huntington, WV 25755, USA E-mail: amina@marshall.edu

Dr Dong M. Shin, Department of Hematology and Medical Oncology, Winship Cancer Institute of Emory University, 1365C Clifton Road, Atlanta, GA 30322, USA

E-mail:dmshin@emory.edu

Key words: apoptosis, epigallocatechin gallate, resveratrol, head and neck cancer, AKT resveratrol has synergistic growth inhibitory effects and provide an important rationale for future clinical development for chemoprevention and treatment of head and neck cancer.

\section{Introduction}

Toxicity is one of the major concerns in using drugs in prevention settings since the recipients of the chemopreventive drugs are normal subjects with high risk for developing cancer. Similarly, most chemotherapy drugs possess severe toxicity and numerous cases require dose reduction or treatment discontinuation. Therefore, those agents with non-toxic or minimal side effects would be ideal candidates as chemopreventive and chemotherapeutic agents. Due to their proven high safety margin through centuries of human consumption as food or as traditional medicines, natural compounds present in fruits, vegetables and spices have drawn special attention for chemoprevention and treatments (1-3). In the last few decades, hundreds of different food-based natural compounds have been investigated for their antitumor potentials. However, only few of the promising compounds have been advanced to clinical trials (4). Resveratrol and epigallocatechin gallate (EGCG) are among the natural compounds that have been tested in multiple clinical trials and their safety profiles have been established through these trials (1).

Resveratrol is a phytoalexin present in grapes, raspberries, blueberries, mulberries and peanuts. Red grapes, particularly the skin, contain higher amounts of resveratrol. Some wines also contain higher amounts of resveratrol $(5,6)$. Several in vitro and in vivo studies suggest that resveratrol may demonstrate promising efficacy against head and neck cancer (7-10). Resveratrol induced apoptosis of human nasopharyngeal cancer cells (7) and squamous cell carcinoma of the head and neck (SCCHN) cells (8), prevented DMBA-induced oral carcinogenesis in a hamster cheek pouch model (9) and selectively induced DNA damage in SCCHN (10). Human studies reported that orally administered resveratrol is reasonably well-tolerated, and demonstrates only mild to moderate side effects at doses of up to $5 \mathrm{~g} /$ day (11-17). However, resveratrol 
undergoes extensive first-pass metabolism in the intestine and liver, and is also a substrate for multi-drug resistant 1 (MDR1) efflux pumps which further limit its intestinal absorption, resulting in a low oral bioavailability $(<1 \%)(18)$.

Green tea prepared from Camellia sinensis is a widely consumed beverage in Southeast Asia and worldwide, and a rich source of antioxidants (19). The anti-tumor and chemopreventive properties of the constituents of green tea have been demonstrated by multiple epidemiological, cell culture and animal model studies $(1,20)$. EGCG, the most abundant catechin (10-50\% of the total catechin) in green tea, has been extensively studied for its chemopreventive, chemotherapeutic and anticarcinogenic effects (20-22). The safety of green tea preparations have been established by several clinical trials (23-26). In a phase II trial in patients with asymptomatic, Rai stage 0 to II chronic lymphocytic leukemia, $31 \%$ patients experienced a reduction of $\geq 20$ in the absolute lymphocyte count at a dose of $2,000 \mathrm{mg}$ polyphenon $\mathrm{E}$ (PPE), b.i.d (24). In another randomized, placebo-controlled phase II trial in patients with high-risk oral premalignant lesions, higher dose levels (750 and 1,000 mg, t.i.d) showed clinical response and histological improvements (25). In a prostate cancer (PCa) chemoprevention trial, green tea PPE did not reduce the likelihood of $\mathrm{PCa}$ in men with baseline high grade prostatic intraepithelial neoplasia and/or atypical small acinar proliferation, although the number of incidents of PCa was less in the treatment arm (27). These trials clearly demonstrate that green tea alone may not be sufficiently active for effective chemoprevention/therapy and the combination of green tea with other natural or synthetic agents has been suggested $(24,25,28)$.

Although the use of drug combinations is a common practice in the chemotherapy field, this approach has not been widely explored using natural agents. Since ideal treatment or chemopreventive strategies should involve non-toxic agents, a combination of natural compounds is a logical approach. However, the challenge is to find ideal combinations of natural compounds which exhibit synergistic antitumor effects or synergistically block carcinogenesis. In the present study, the antitumor effects of the combination of resveratrol and EGCG against SCCHN cell lines were investigated via apoptosis assay, western blotting, IHC and using an in vivo xenograft model in nude mice.

\section{Materials and methods}

Cell lines. Cell lines used in the present study have been previously described $(29,30)$. Tu212, a cell line of hypopharyngeal origin, was kindly provided by Dr Gary L. Clayman (University of Texas M.D. Anderson Cancer Center, Houston, TX, USA). MDA686TU (Tu686) and MSK-Leuk1 (MSK) were procured from Dr Peter G. Sacks (New York University College of Dentistry, New York, NY, USA) in 2014 and 2012, respectively. Tu686 was established from primary tongue cancer. The head and neck premalignant cell line MSK-Leuk1 (MSK) was established from a dysplastic leukoplakia lesion adjacent to a squamous cell carcinoma of the tongue and was maintained in keratinocyte basal media. SqCCY1 was established from a squamous carcinoma of the buccal mucosa in Dr Sartorelli's laboratory (Yale University, New Haven, CT,
USA) (31) and was obtained from Dr Shi-Yong Sun at Emory University (Atlanta, GA, USA) in 2012. All cell lines were authenticated through genotyping [short tandem repeats (STR) profiling]. SCCHN cell lines were maintained in DMEM/F12 (Corning, Inc.) medium supplemented with $10 \%$ heat-inactivated fetal bovine serum (Gibco; Thermo Fisher Scientific, Inc.) in a $37^{\circ} \mathrm{C}$ and $5 \% \mathrm{CO}_{2}$ humidified incubator. STR profiles of these cell lines matched with those published in Clinical Cancer Research 2011 (32), suggesting that Tu212 is identical with some other SCCHN cell lines but other cell lines are unique.

Annexin V-phycoerythrin staining for apoptosis. MDA686TU, Tu212 and SqCCy1 cells $\left(1.5 \times 10^{5}\right.$ cells $/ 6-\mathrm{cm}$ plate $)$ were treated with various concentrations of EGCG (30-200 $\mu \mathrm{M}$; Sigma-Aldrich; Merck KGaA), resveratrol (10-70 $\mu \mathrm{M}$; Sigma-Aldrich; Merck KGaA) and their combination (EGCG: 30-80 $\mu \mathrm{M}$; resveratrol: $10-20 \mu \mathrm{M})$ for $72 \mathrm{~h}$, then trypsinized and washed in cold 1X PBS (Corning, Inc.). The cells were then resuspended in 1X Annexin $\mathrm{V}$ binding buffer (BD Biosciences), and stained with Annexin V-phycoerythrin (Annexin V-PE; BD Biosciences) and 7-AAD (BD Biosciences) for $15 \mathrm{~min}$ at room temperature. The stained samples were analyzed using a fluorescence-activated cell sorting caliber bench-top flow cytometer (BD Biosciences). FlowJo_v10.6.0_CL software (Tree Star, Inc.) was used for apoptosis analysis. Total apoptosis was considered the sum of early and late stage apoptosis. Combination Index (CI) values were calculated by using CalcuSyn 2.11 software (Biosoft).

Western blot analysis. Whole cell lysates were extracted from Tu212, MDA686TU and SqCCy1 cells using lysis buffer (for $200 \mathrm{ml}: 10 \mathrm{ml} 1 \mathrm{M}$ Tris HCl, $6 \mathrm{ml} 5 \mathrm{M} \mathrm{NaCl}, 200 \mu \mathrm{l}$ $20 \%$ sodium azide, $1 \mathrm{~g}$ sodium deoxycholate, $4 \mathrm{ml}$ Igepal, $2 \mathrm{ml}$ $20 \%$ SDS) and the protein concentration of each sample was determined by Quick Start Bradford protein assay kit (Bio-Rad Laboratories, Inc.). Equal amounts of protein (15-20 $\mu \mathrm{g})$ from each sample were separated on 10 or $12 \%$ sodium dodecyl sulfate-polyacrylamide gel electrophoresis (SDS-PAGE). The proteins were transferred to polyvinylidene difluoride (PVDF) membranes (EMD Millipore), blocked in 5\% non-fat skimmed milk for $2 \mathrm{~h}$ at room temperature and incubated with appropriately diluted specific primary antibodies overnight at $4^{\circ} \mathrm{C}$, followed by incubation with a secondary antibody for $1 \mathrm{~h}$ at room temperature. Mouse anti- $\beta$-actin (Sigma-Aldrich; Merck KGaA) antibody was used as a sample loading control. Immunostained protein bands were detected with an enhanced chemiluminescence kit (Pierce; Thermo Fisher Scientific, Inc.). Phosphorylated (p)-AKT (product no. 4060), AKT (product no. 4685), PARP (product no. 9542), cleaved caspase-3 (product no. 9661), p-mTOR (product no. 5536), p-S6 (product no. 4858), S6 (product no. 2317), p-4EBP-1 (product no. 2855), 4E-BP1 (product no. 9644) primary antibodies were purchased from Cell Signaling Technology, Inc. and Mcl-1 (cat. no. sc-377487) and survivin (cat. no. sc-374616) from Santa Cruz Biotechnology, Inc. Anti-mouse (product no. W402B) and anti-rabbit IgG (product no. W401B) were purchased from Promega Corporation. All primary antibodies were diluted to $1: 1,000$ and secondary antibodies to $1: 10,000$ in 5\% non-fat skimmed milk. 
In vivo xenograft model. The animal experiments were approved by the Institutional Animal Care and Use Committee of Emory University (DAR-2002630-050517BN). A total of 20 female nude mice (athymic nu/nu; Taconic Biosciences, Inc.), aged 4-6 weeks (weight, $20 \mathrm{~g}$ ), were used in the present study. Mice were maintained on Alpha-Dry bedding in temperature $\left(22 \pm 2^{\circ} \mathrm{C}\right)$ and humidity $(30-50 \%)$ controlled rooms with a 12-h light/dark cycle. Rodent Chow No. 5010 (LabDiet) and autoclaved water were provided ad libitum. After adaptation for a few days in the new environment, the mice were subcutaneously injected with $2.5 \times 10^{6} \mathrm{Tu} 212$ cells into the right flank. After approximately a week when visible tumors had formed, the mice were randomly divided into four groups ( $\mathrm{n}=5$ in each group). Each mouse was orally gavaged with vehicle control ( $0.5 \%$ carboxymethyl cellulose), EGCG $(125 \mathrm{mg} / \mathrm{kg}$ in water), resveratrol $(30 \mathrm{mg} / \mathrm{kg}$ in $0.5 \%$ carboxymethyl cellulose) or a combination of EGCG $(125 \mathrm{mg} / \mathrm{kg})$ and resveratrol $(30 \mathrm{mg} / \mathrm{kg}) 5$ days a week. The tumor size (larger diameter and smaller diameter) was measured 2 times a week using a digital caliper. The tumor volume was calculated using the formula: $\mathrm{V}=\pi / 6 \mathrm{x}$ larger diameter $\mathrm{x}$ (smaller diameter $)^{2}$. Growth curves were plotted using the average tumor volume within each experimental group at the set time-points $(0,4,6,8,11,14,20,25$ and 28 days). At the end of the study, mice were euthanized with $\mathrm{CO}_{2}$ from a gas tank (flow rate: $30 \%$ chamber vol $/ \mathrm{min}$ ); the mice were placed in a euthanasia chamber to allow visualization during the procedure. Each mouse was observed for lack of respiration and faded eye color. $\mathrm{CO}_{2}$ flow was maintained for a minimum of $1 \mathrm{~min}$ after respiration ceased and sacrifice was confirmed through fixed and dilated pupils.

Immunohistochemistry and TUNEL assay. Formalin-fixed (Z-Fix; 10\% aqueous buffered zinc formalin; Anatech Ltd.; overnight at room temperature) paraffin-embedded (FFPE) xenograft tumor slides (3 microns) were deparaffinized with xylene and dehydrated with a series of alcohol treatments. The slides were incubated with $3 \%$ hydrogen peroxide in water to block endogenous peroxidase activity and subjected to antigen retrieval using $1 \mathrm{X}$ citrate buffer $(\mathrm{pH}$ 6.0) in a microwave followed by cooling at room temperature. After washing, the slides were incubated with a blocking buffer provided in a kit (Vectastain Kit cat. no. PK7800; Vector Laboratories, Inc.) followed by incubation with primary antibodies Ki67 (1:400 dilution; product code ab15580; Abcam), p-AKT (1:50 dilution, product no. 4060), p-S6 (1:50 dilution; product no. 4858) and p-4EBP1 (1:100 dilution; product no. 2855) (all from Cell Signaling Technology, Inc.) overnight in a refrigerator. The slides were washed and incubated with a ready to use secondary antibody provided with the kit for $30 \mathrm{~min}$ at room temperature followed by chromogen visualization using 3,3-diaminobenzideine (DAB) provided with the kit. The slides were counterstained with hematoxylin for $1 \mathrm{~min}$ at room temperature for nuclei visualization and five random areas were selected for analysis. The TUNEL assay was performed by immunofluorescence using the same specimens as aforementioned, following the procedure provided by the manufacturer (cat. no. C10617; Life Technologies; Thermo Fisher Scientific, Inc.). To analyze the assay results, the total number of cells and the positive number of cells in the same area were counted for five random areas; the result was presented as an average ratio of the positive number of cells out of the total number of cells. The magnification was $\mathrm{x} 200$ for all IHC images.

Statistical analysis. The effect of the combination of EGCG and resveratrol on the inhibition of tumor growth was assessed through in vivo and in vitro experiments. For in vivo data analyses, the expression of TUNEL and Ki67 among the treatment groups EGCG, resveratrol, combination of EGCG and resveratrol, and control was compared by using two-way ANOVA after adjusting the area from where the cells were obtained. Bonferroni adjustment was performed for multiple comparisons between EGCG, resveratrol, combination, and control. For in vivo tumor growth analyses, a mixed effect model with random subject effect was used by accounting the repeated outcomes over time. The data were collected repeatedly 9 times from the same mouse and the tumor volume was measured. In the model, compound symmetry covariance structure based on minimum AIC criteria was used. The tumor volume was compared between treatment groups EGCG, resveratrol, and combination vs. control. The comparisons between the control and treatment groups were adjusted using Bonferroni correction. All statistical analyses were performed using SAS version 9.4 (SAS Institute). Results were also validated by cross-checking with other software, $\mathrm{R}$ (version 3.6.2; The R Foundation for Statistical Computing) (33). The results were considered statistically significant when the $\mathrm{P}$-values were $<0.05$.

\section{Results}

Synergistic apoptosis induced by the combination of EGCG and resveratrol. It has been previously reported by our research group that the combination of two natural compounds, EGCG and luteolin, induced enhanced antitumor effects against both lung and head and neck cancers both in vitro and in vivo (29). In an effort to identify new combinations of natural compounds with synergistic apoptosis, the combination of EGCG and resveratrol, a combination never explored in vivo and in in head and neck cancer were studied. First, the sensitivity of three SCCHN cell lines to different doses of EGCG and resveratrol were examined, and a dose-dependent apoptosis induced by the compounds as single agents was revealed (Fig. 1A-C). Next, induction of apoptosis using low doses of EGCG in combination with two doses of resveratrol was examined. As revealed in Fig. 1A-C right panels, the combination treatment significantly increased apoptosis although single agents induced markedly little apoptosis at these doses. In order to confirm synergistic apoptotic effects, the apoptosis data was analyzed using CalcuSyn software and it was revealed that combination of the two agents induced highly synergistic apoptosis as indicated by combination index values $<1$ (Table I). Synergistic apoptosis was also supported by cleavage of PARP and caspase-3 (Fig. 2A). Combination of the two agents at lower doses also completely eradicated tumors cells (Fig. 2B). The efficacy of the combination of EGCG and resveratrol against a premalignant cell line, MSK-LEUK1, was next tested. As revealed in Fig. 2C, the premalignant cell line was sensitive to the combination of considerably lower doses of each agent, suggesting that the combination of EGCG and resveratrol is also suitable for chemoprevention. 

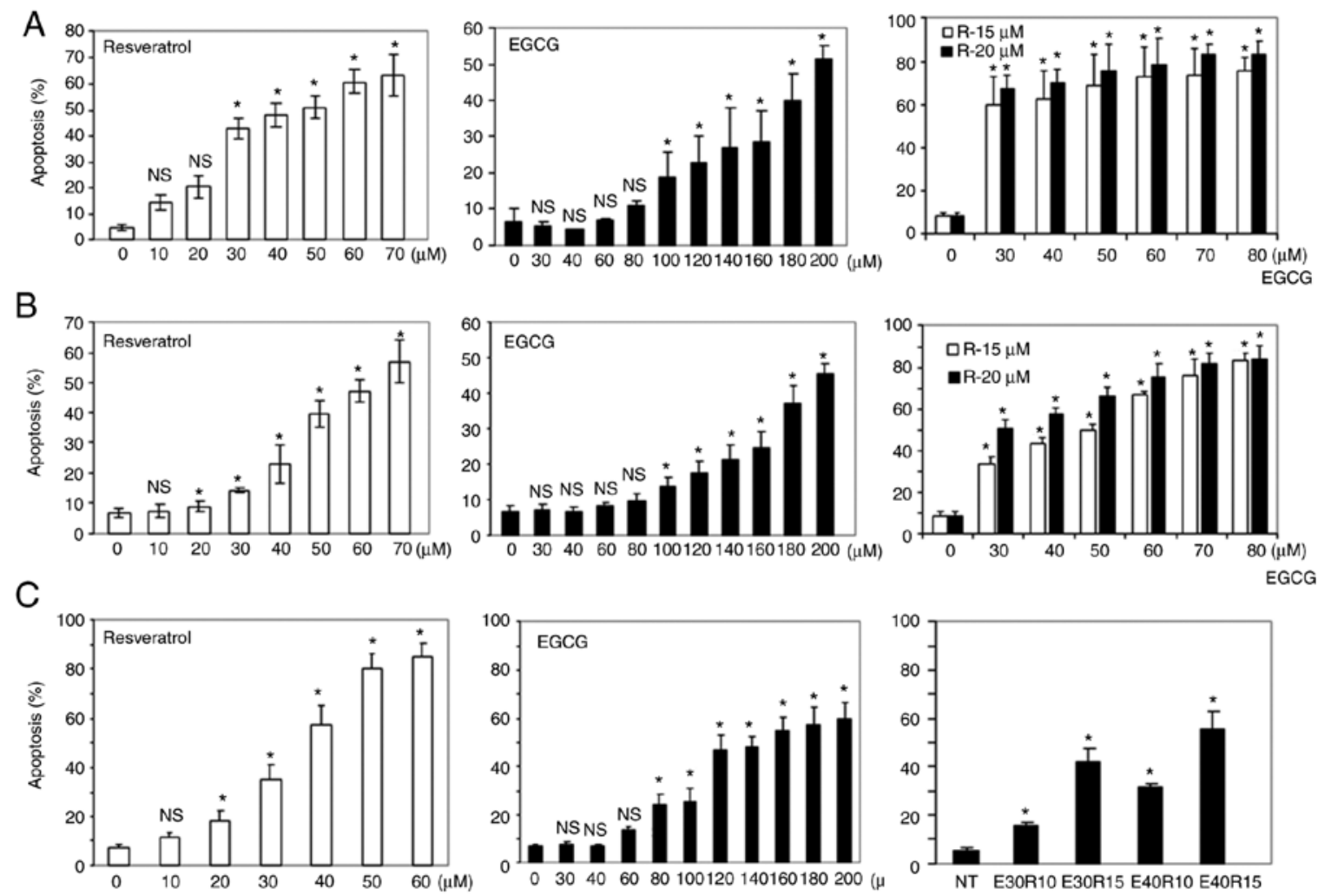

Figure 1. Induction of apoptosis by the combination of EGCG and resveratrol. (A) SqCCy1, (B) MDA686Tu and (C) Tu212 cells were treated with various doses of EGCG, resveratrol and their combination as indicated for $72 \mathrm{~h}$. Apoptosis was measured by Annexin V-PE staining. Total apoptosis was determined by combining early and late apoptotic cells. Results from triplicate treatments are presented with standard deviations as error bars. ${ }^{*} \mathrm{P}<0.05$ indicates statistically significant increase in apoptosis when compared with the corrsponding untreated control. EGCG, epigallocatechin gallate. X-axes indicate concentrations $(\mu \mathrm{M})$ of resveratrol (left-hand panels), EGCG (middle panels). Right panels: Combination of EGCG and resveratrol as indicated in the images.

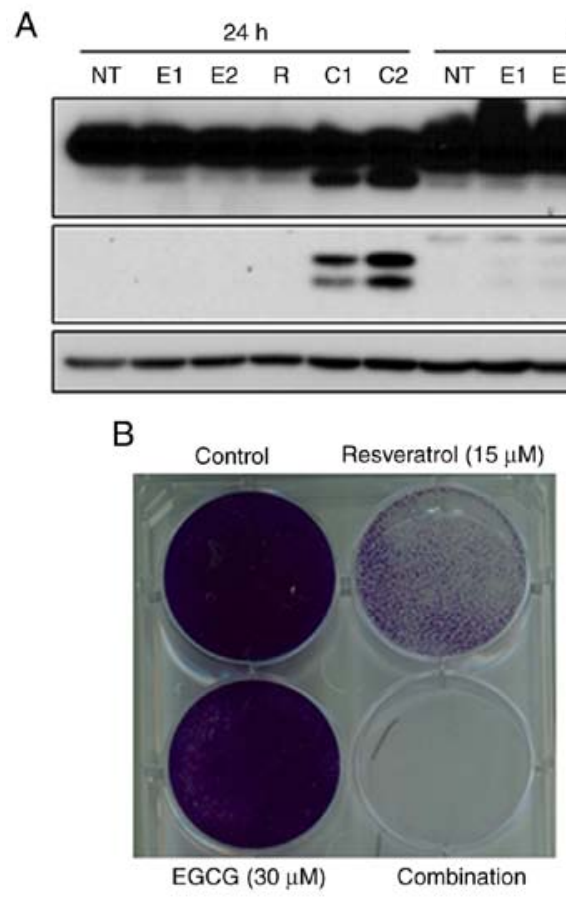

$\mathrm{E} 1=30 \mu \mathrm{M}$ EGCG
$\mathrm{E} 2=40 \mu \mathrm{M}$ EGCG
$\mathrm{R}=15 \mu \mathrm{M}$ resveratrol
$\mathrm{C} 1=\mathrm{E} 1+\mathrm{R}$
$\mathrm{C} 2=\mathrm{E} 2+\mathrm{R}$

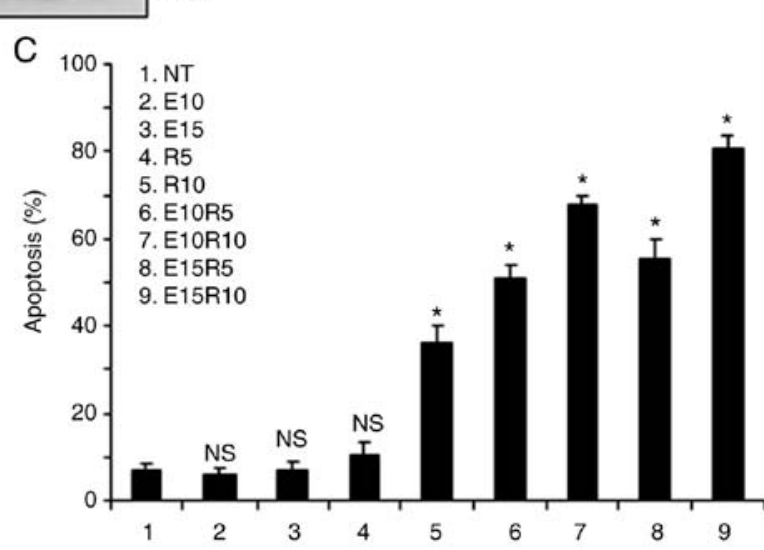

Figure 2. Combination of EGCG and resveratrol is suitable for chemoprevention. (A) Tu212 cells were treated with the indicated doses of EGCG, resveratrol and their combination. Total cell lysates were used to assess the expression of PARP and cleaved caspase-3. Findings were confirmed by 2 or 3 repeats with almost similar experimental design (variation in dose and treatment time). Representative blots are presented. (B) Tu212 cells were treated with $30 \mu \mathrm{M} \mathrm{EGCG,}$ $15 \mu \mathrm{M}$ resveratrol and their combination for two weeks. Plates were stained with $0.5 \%$ crystal violet. (C) MSK-Leuk1 (premalignant) cells were treated with different doses of EGCG, resveratrol and their combination for $72 \mathrm{~h}$. Apoptosis was measured by Annexin V-PE staining from triplicate treatments. Error bars represent standard deviations. " $\mathrm{P}<0.05$ indicates statistically significant increase in apoptosis when compared with the corresponding untreated control. EGCG, epigallocatechin gallate. 
Table I. Combination index at different combinations.

\begin{tabular}{|c|c|c|c|}
\hline \multirow[b]{2}{*}{ Cell line } & \multirow[b]{2}{*}{$\begin{array}{c}\text { EGCG } \\
(\mu \mathrm{M})\end{array}$} & \multicolumn{2}{|c|}{ Combination Index } \\
\hline & & $\begin{array}{l}\text { Resveratrol } \\
\quad(15 \mu 1)\end{array}$ & $\begin{array}{c}\text { Resveratrol } \\
\quad(20 \mu \mathrm{l})\end{array}$ \\
\hline \multirow[t]{7}{*}{$\mathrm{Tu} 212$} & 30 & 0.93 & 0.73 \\
\hline & 35 & 0.68 & 0.63 \\
\hline & 40 & 0.66 & 0.62 \\
\hline & 45 & 0.62 & 0.68 \\
\hline & 50 & 0.63 & 0.74 \\
\hline & 55 & 0.61 & 0.43 \\
\hline & 60 & 0.59 & 0.47 \\
\hline \multirow[t]{6}{*}{ SqCCy1 } & 30 & 0.41 & 0.42 \\
\hline & 40 & 0.43 & 0.44 \\
\hline & 50 & 0.43 & 0.43 \\
\hline & 60 & 0.47 & 0.44 \\
\hline & 70 & 0.49 & 0.43 \\
\hline & 80 & 0.49 & 0.44 \\
\hline \multirow[t]{6}{*}{ MDA686TU } & 30 & 0.49 & 0.46 \\
\hline & 40 & 0.47 & 0.46 \\
\hline & 50 & 0.48 & 0.46 \\
\hline & 60 & 0.43 & 0.44 \\
\hline & 70 & 0.41 & 0.43 \\
\hline & 80 & 0.41 & 0.45 \\
\hline
\end{tabular}

Tu212, SqCCy1 and MDA686TU cells were treated with the indicated doses of EGCG, resveratrol and their combination and apoptosis was assessed after $72 \mathrm{~h}$. Combination index values were calculated using CalcuSyn software. EGCG, epigallocatechin gallate.

Inhibition of growth of xenograft tumors in nude mice by the combination of EGCG and resveratrol. The in vivo antitumor efficacy of the combined treatment with EGCG and resveratrol was investigated in xenografted mice bearing Tu212 cells, an established SCCHN cell line known to generate tumor in nude mice (34-36). Each group consisted of five mice. Although EGCG and resveratrol as single agents had minimal effect on tumor growth, their combination inhibited tumor growth to a statistically significant level $(\mathrm{P}=0.026)$ (Fig. 3A) compared to single-agent resveratrol $(\mathrm{P}=0.182)$ and EGCG $(\mathrm{P}=0.130)$. The tumor weight at the end of the study was also assessed. Combination of the two agents also significantly inhibited tumor weight (Fig. 3B). Images of tumors obtained after sacrifice are presented in Fig. 3C. To confirm the aforementioned results in tissue levels, tumor tissues were also stained for the expression of Ki67 (proliferation marker) and TUNEL (apoptosis marker). Ki67- and TUNEL-positive cells were quantified and it was revealed that the combination of EGCG and resveratrol significantly inhibited Ki67-positive cells (Fig. 3D and E). The number of TUNEL-positive cells was increased similarly by EGCG, resveratrol and their combination (Fig. 3D and F). These in vivo data thus confirmed the in vitro results.

Inhibition of AKT-mTOR signaling by the combination of EGCG and resveratrol. Activation of AKT and its target
mTOR, has been observed in $>80 \%$ of SCCHN lesions $(37,38)$. The effect of EGCG, resveratrol and their combination on AKT-mTOR pathway markers was next examined. As revealed in Fig. 4A and B, treatment of SCCHN cells with EGCG, resveratrol and their combination markedly inhibited p-AKT, p-mTOR and downstream p-S6 and p-4E-BP1 in vitro. In order to associate in vitro data with in vivo biomarker modulation, xenografted tumor tissues were stained for the expression of p-AKT, p-S6 and p-4E-BP1 (Fig. 4C). As revealed in Fig. 4C, treatment with EGCG, resveratrol and their combination inhibited the expression of p-AKT. On the other hand, only the combination of EGCG and resveratrol strongly inhibited the expression of $\mathrm{p}-\mathrm{S} 6$. In the case of $\mathrm{p}-4 \mathrm{E}-\mathrm{BP} 1$, treatment with EGCG, resveratrol and their combination inhibited the expression of p-4E-BP1. Combination of the two agents also significantly inhibited the expression of $\mathrm{p}-4 \mathrm{E}-\mathrm{BP} 1$ as compared with single agent treatments. To confirm the role of inhibition of the AKT pathway in apoptosis, MDA686TU cells overexpressing constitutively active (CA-AKT) were used (39). As revealed in Fig. 5A, overexpression of CA-AKT increased the basal level of S6 phosphorylation more strongly than 4E-BP1 phosphorylation. Overexpression of CA-AKT also significantly protected cells from apoptosis induced by the combination of EGCG and resveratrol, suggesting that inhibition of AKT-mTOR signaling is required for the induction of apoptosis by the combination of the two agents. It was also revealed that the combination of the two agents more strongly inhibited the expression of the survival proteins Mcl-1 and survivin as compared to single-agent treatment (Fig. 6).

\section{Discussion}

To date, the most successful drugs for the cure of cancer and eradication of tumor cells from the body are cytotoxic chemotherapy drugs, molecularly targeted agents and immunotherapy drugs $(40,41)$. These agents are capable of inducing apoptosis, although most have significant toxicities. In contrast, cytostatic drugs induce growth arrest without eliminating cancer cells from the body, thus the acquisition of resistance is a common phenomenon $(42,43)$. While relatively non-toxic, low bioavailability limits the success of food-derived natural compounds in chemoprevention and treatment of cancers $(1,3)$, combinatorial approaches with synergistic effects may be a solution to improve the efficacy of food-derived natural agents. In the present study, apoptosis using the combination of two natural compounds, EGCG and resveratrol was investigated. The present data clearly demonstrated that high concentrations are required for the induction of significant apoptosis when these agents are used alone. Due to the low bioavailability of these compounds, it may not be possible to achieve such high concentrations in vivo that will be effective for tumor regression. In contrast, combination of the two agents induced synergistic apoptosis at relatively low doses. The in vitro data is supported by in vivo xenograft data revealing that only the combination of two agents inhibited tumor growth (reduction of tumor volume and tumor weight, and expression of Ki67) with a statistically significant P-value. The in vitro data also suggest that premalignant cells (MSK-LEUK1) are markedly more sensitive to the combination of the two agents (i.e, a lower dose was required for similar effects), and thus this combination 


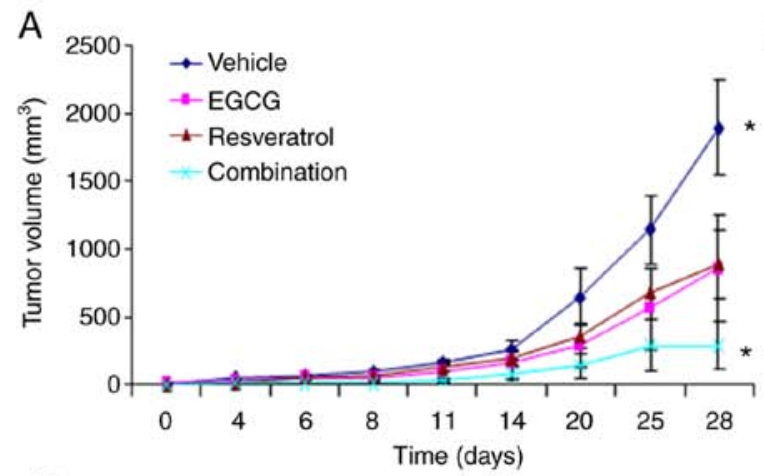

C
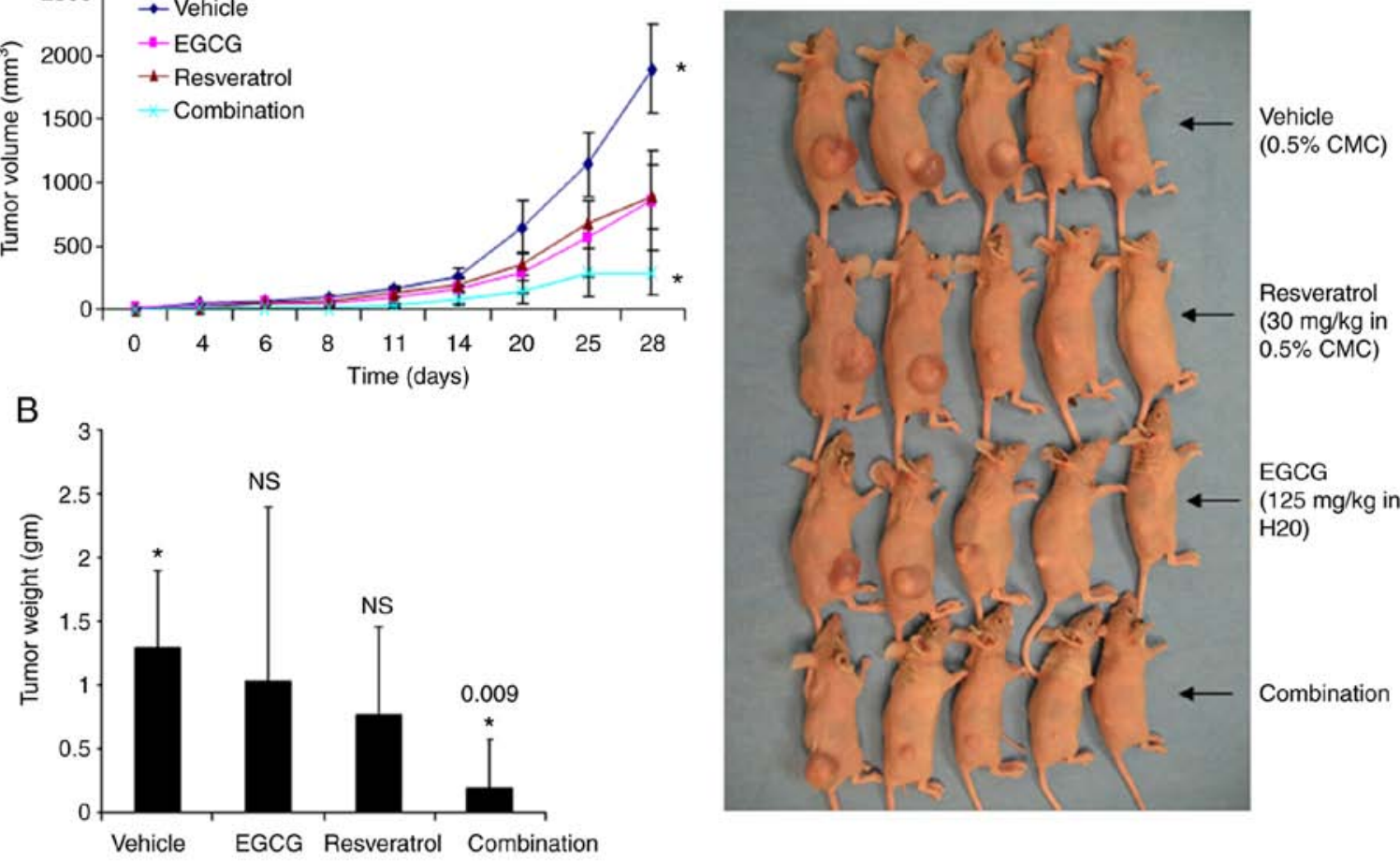

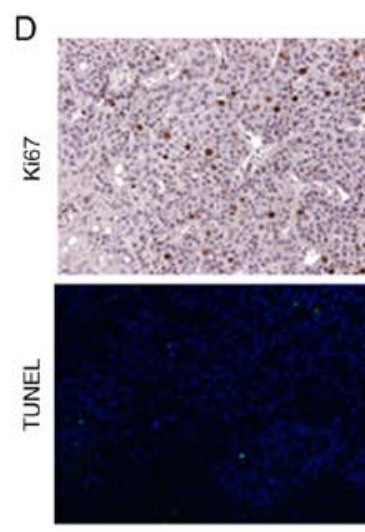

Vehicle

E

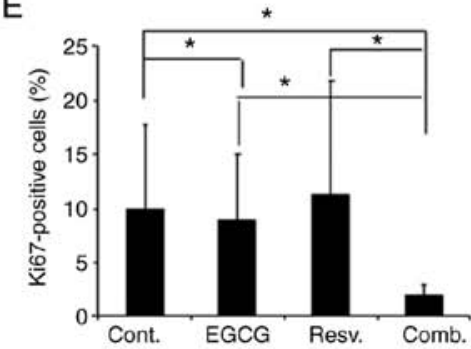

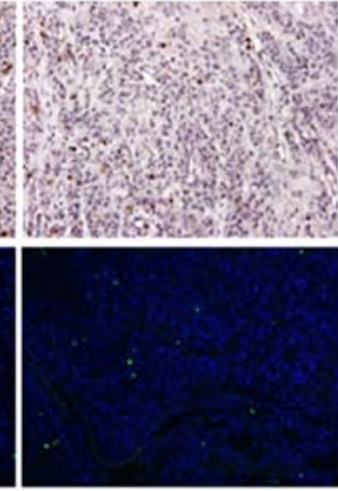

EGCG

Comb.
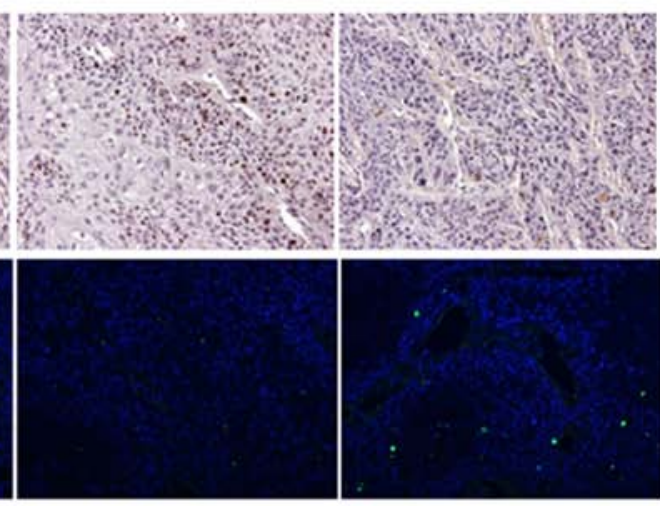

Resveratrol

Combination

$\mathrm{F}$

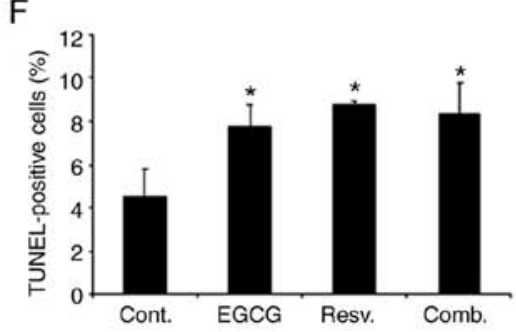

Figure 3. Inhibition of in vivo tumor growth by the combination of EGCG and resveratrol in nude mice. (A) Four groups of animals were orally gavaged with vehicle control, EGCG, resveratrol, or their combination as described in Materials and methods. Growth curves were obtained for xenografted tumors. $\mathrm{Tu} 212$ tumor growth was significantly inhibited only in the combination group as compared with the control (P=0.026). Other comparisons were insignificant $(\mathrm{P}>0.05)$. (B) Tumors were weighed at the end of the experiment. Similar to tumor volumes, tumor weights were also significantly inhibited only in the combination group as compared with the control. (C) Images of tumor-bearing mice at the end of the study. (D) Ki67 and TUNEL expression detected in xenograft tissues by immunohistochemical analysis. Representative images are presented from each group (magnification, x200). (E and F) Quantification of Ki67 and TUNEL staining. " $\mathrm{P}<0.05$ indicates a statistically significant difference. EGCG, epigallocatechin gallate.

may also be suitable in chemoprevention settings. To the best of our knowledge, this is the first such study reporting in vitro and in vivo antitumor effects as well as synergistic apoptosis of the combination of two natural compounds EGCG and resveratrol against head and neck cancer.
The mechanism of synergistic apoptosis induced by the combination was also explored. Anti-apoptotic Bcl-2 proteins including Bcl-2, Bcl-xL and Mcl-1 serve as mitochondrial gatekeepers and maintain mitochondrial membrane integrity (44). Inhibition of the expression of these anti-apoptotic 

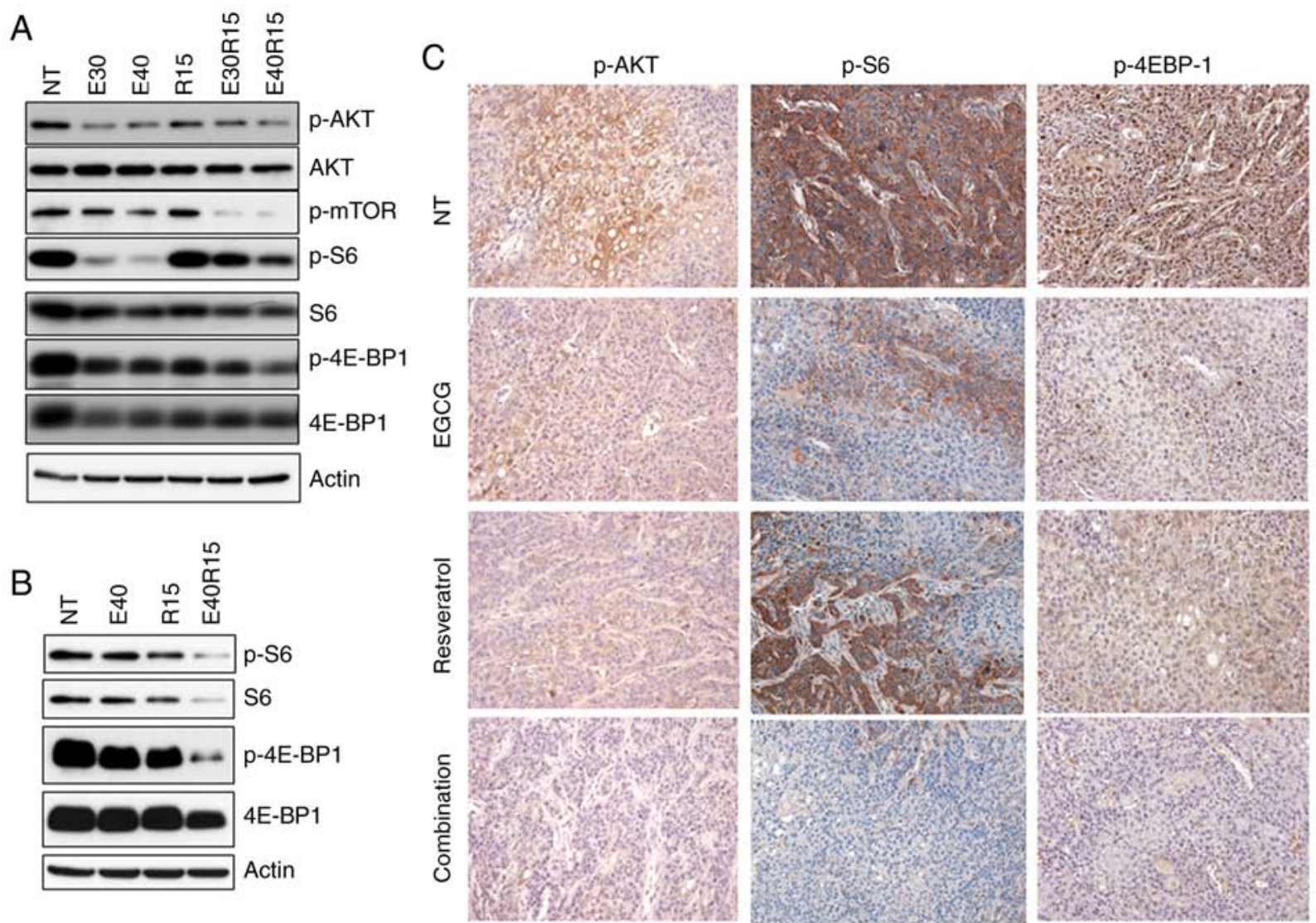

Figure 4. Inhibition of AKT-mTOR signaling by the combination of EGCG and resveratrol. (A) Tu212 and (B) SqCCy1 cells were treated with the indicated doses of EGCG, resveratrol or their combination for $24 \mathrm{~h}$. Expression of p-AKT, p-mTOR, p-S6 and p-4E-BP1 was examined by western blotting in total cell lysates. Findings for A and B were confirmed by 2 or 3 repeats with almost similar experimental design (variation in dose and treatment time). Representative blots are presented. (C) Xenografted tumor tissues were stained for the expression of p-AKT, p-S6 and p-4E-BP1 by immunohistochemistry. Representative images from each group are presented. NT, no treatment; $p$, phosphorylated.
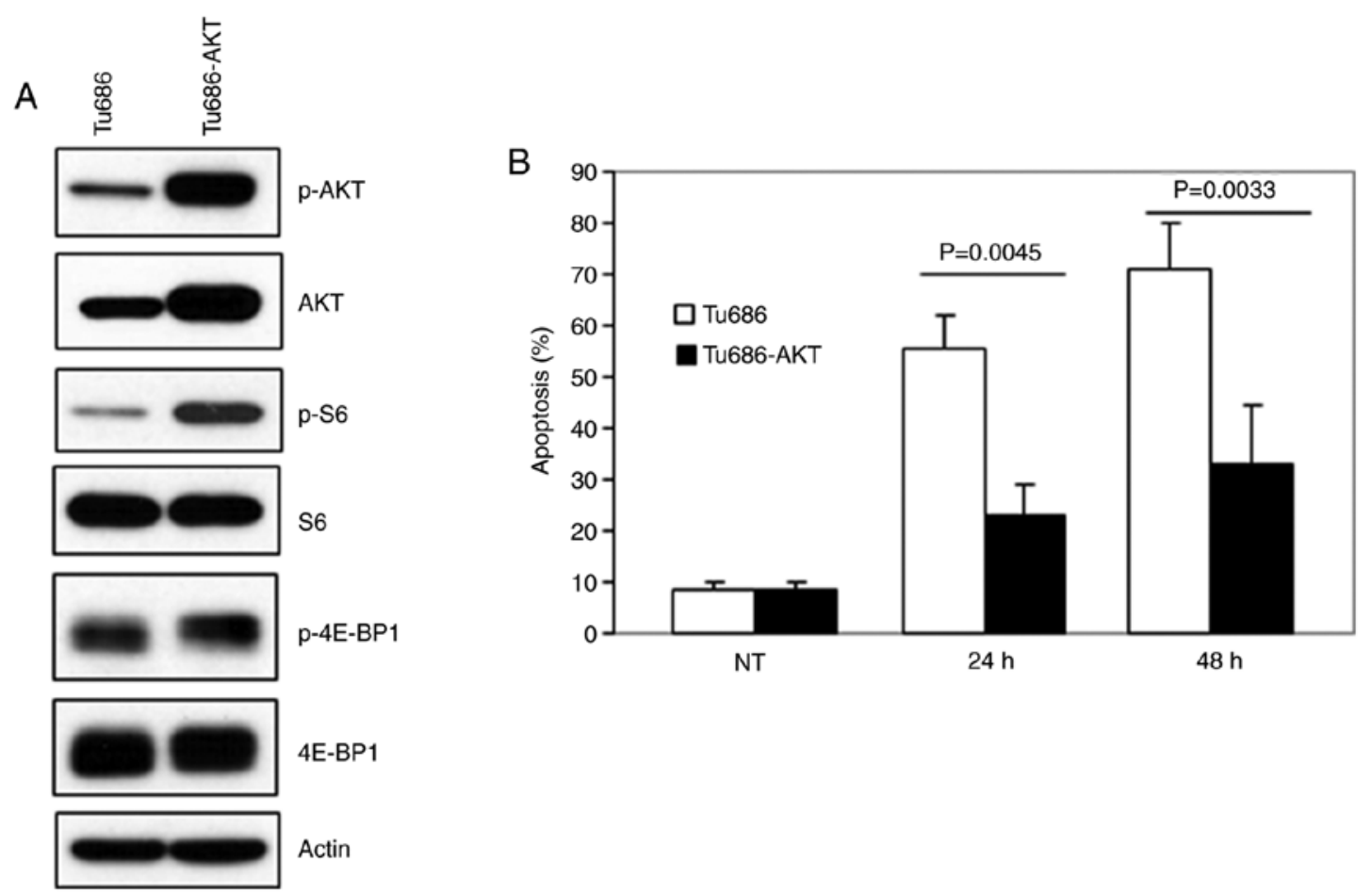

Figure 5. Inhibition of AKT-mTOR signaling is required for apoptosis induced by the combination of EGCG and resveratrol. (A) MDA686TU cells were transduced with constitutively active AKT and expression of p-AKT, p-S6 and p-4E-BP1 was examined in whole cell lysates. (B) Non-transduced and transduced cells were treated with the combination of EGCG and resveratrol and apoptosis was measured after $72 \mathrm{~h}$. Averages of triplicate treatments were graphed with standard deviations as error bars. EGCG, epigallocatechin gallate; p, phosphorylated; NT, no treatment. 

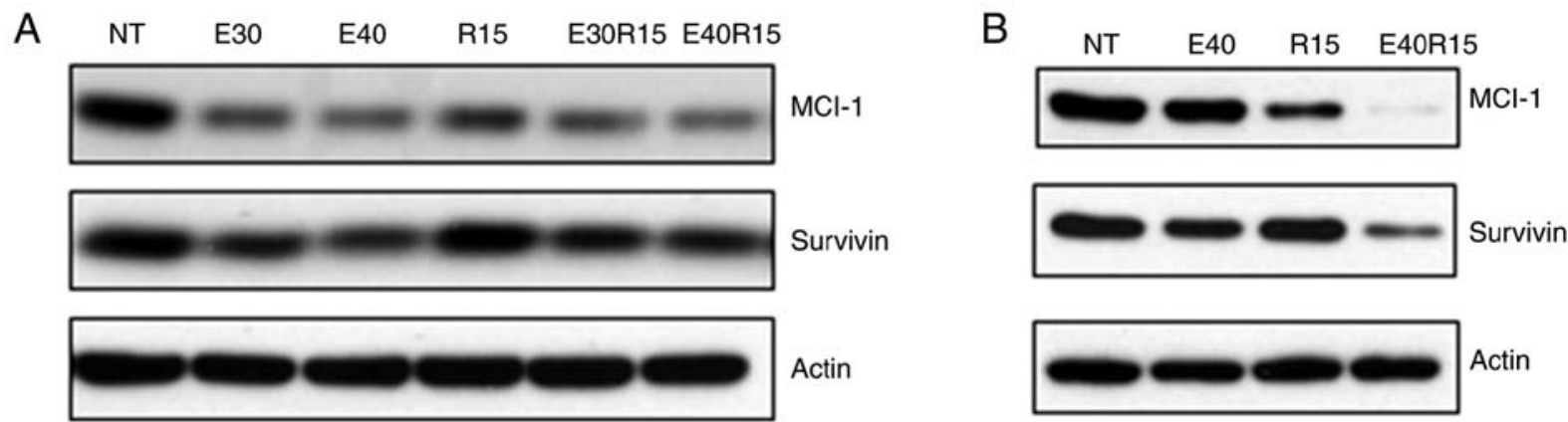

Figure 6. Inhibition of Bcl-2 proteins and survivin. (A) Tu212 and (B) SqCCy1 cells were treated with the indicated doses of EGCG, resveratrol and their combination. Expression of MCL-1 and survivin was examined in total cell lysates. Findings were confirmed by 2 or 3 repeats with almost similar experimental design (variation in dose and treatment time). Representative blots are presented. EGCG or E, epigallocatechin gallate; R, resveratrol.

Bcl-2 proteins favors apoptosis by inhibiting mitochondrial membrane potential, allowing release of cytochrome $c$ in the cytoplasm to activate the apoptotic cascade. Survivin, a member of the inhibitors of apoptosis (IAP) family, also protects cells from apoptosis. Multiple cell survival pathways are associated with the pathogenesis of SCCHN. In the present study, it was revealed that treatment of cells with EGCG, resveratrol and their combination inhibited the expression of Mcl-1 and survivin.

Genome-wide sequence analyses of SCCHN have identified the most frequent genetic alterations in these diseases $(37,38)$. Several mutations were identified in the phosphoinositide 3-kinase (PI3K)/mammalian target of rapamycin (mTOR) pathway, including PIK3CA (8-10\%), TSC1/2 (5-8\%), and PTEN (5-10\%), all of which result in PI3K/AKT/mTOR pathway activation $(37,38,45-48)$. Activation of AKT and its target mTOR has been observed in $>80 \%$ of SCCHN lesions, implicating the AKT/mTOR pathway as an excellent target in SCCHN. The present data demonstrated that treatment with EGCG, resveratrol or their combination inhibited p-AKT, p-mTOR and downstream p-S6 and p-4E-BP1 both in vitro and in vivo. Notably, overexpression of constitutively active AKT (CA-AKT) which rescues p-AKT and downstream mTOR pathways, significantly protected cells suggesting the critical role of p-AKT inhibition in apoptosis induced by the combination of EGCG and resveratrol. A number of previous studies suggest that inhibition of mTOR pathways successfully prevents the development of SCCHN in 4NQO-induced and genetic mouse models (49-51). It was also previously reported by our research group that inhibition of pS6, a downstream target of mTOR signaling was correlated with clinical response in our celecoxib/erlotinib trial of SCCHN chemoprevention (52). Short noncoding RNAs including microRNAs (also known as miRNAs or miRs) modulate gene expression at the post transcriptional level via sequence-specific interactions with the cognate mRNA targets (53). miRNAs regulate gene silencing through both degradations of the mRNA and inhibition of translation and play crucial roles in carcinogenesis as well as in regulating cell growth and survival (54). Accumulated evidence suggests that both EGCG and resveratrol modulate the expression of miRs. For example, EGCG suppressed growth and tumorigenicity in breast cancer cells by downregulation of miR-25 (55). Another study demonstrated that EGCG modulated the expression of miR-203, miR-125b, miR-210 and miR-29a in cervical carcinoma cell lines (56). Similarly, triacetyl resveratrol upregulated miR-200 in pancreatic cancer cell lines (57). It is possible that the combination of EGCG and resveratrol inhibits tumor cell growth by modulating the expression of miRs, which needs to be confirmed through future studies.

Unique identitity of cell lines used for evaluting anticancer drugs is critical for efficacy studies as well as elucidating their mechanism of action. Misidentification and cross contamination is unfortunately common and can generate misleading results. One of the limitations of the present study is the use of the Tu212 cell line. The unique identity of this cell line is under question since some other SCCHN cell lines used by the head and neck community have a similar STR profile. Multiple cell lines were used to confirm the present results. All other cell lines were confirmed by STR profiling to have a unique identity. In conclusion, the present study has identified that the combination of EGCG and resveratrol induced synergistic apoptosis and inhibited SCCHN xenograft growth in vivo, and explored the potential mechanisms. The present results have high translational significance and will pave the way for further clinical development of the combination of EGCG and resveratrol, particularly for the chemoprevention of head and neck cancers and other malignacies.

\section{Acknowledgements}

The authors thank Dr Anthea Hammond (Scientific Writer, Department of Hematology and Medical Oncology, Winship Cancer Institute of Emory University) for editing the manuscript.

\section{Funding}

The present study was supported by the start-up funding from Winship Cancer Institute of Emory University (ARA), the Faculty Research Support grant from Marshall University School of Pharmacy (ARA), the WV-INBRE grant (no. P20GM103434) (ARA) and the Head and Neck Cancer SPORE (grant no. P50CA128613) (DMS and GC).

\section{Availability of data and materials}

The datasets used during the present study are available from the corresponding author upon reasonable request. 


\section{Authors' contributions}

All the authors contributed to the conception and design of the study. ARMRA, DW and SN acquired the data. ARMRA, SN, RL, ZGC and DMS analyzed and interpreted the data. ARMRA, SN, RL drafted the manuscript. ARMRA, DW, ZGC and DMS critically revised the manuscript for important intellectual content. All authors read and approved the final version of the manuscript.

\section{Ethics approval and consent to participate}

The animal experiments were approved by the Institutional Animal Care and Use Committee of Emory University (DAR-2002630-050517BN).

\section{Patient consent for publication}

Not applicable.

\section{Competing interests}

The authors declare that they have no competing interests.

\section{References}

1. Amin AR, Kucuk O, Khuri FR and Shin DM: Perspectives for cancer prevention with natural compounds. J Clin Oncol 27: 2712-2725, 2009.

2. Rahman MA, Amin AR and Shin DM: Chemopreventive potential of natural compounds in head and neck cancer. Nutr Cancer 62: 973-987, 2010.

3. Gullett NP, Ruhul AR Amin AR, Bayraktar S, Pezzuto JM, Shin DM, Khuri FR, Aggarwal BB, Surh YJ and Kucuk O: Cancer prevention with natural compounds. Semin Oncol 37: 258-281, 2010

4. Park W, Amin AR, Chen ZG and Shin DM: New perspectives of curcumin in cancer prevention. Cancer Prev Res (Phila) 6: 387-400, 2013.

5. Burns J, Yokota T, Ashihara H, Lean MEJ and Crozier A: Plant foods and herbal sources of resveratrol. J Agric Food Chem 50: 3337-3340, 2002.

6. Cione E, Torre CL, Cannataro R, Caroleo MC, Plastina P and Gallelli L: Quercetin, epigallocatechin gallate, curcumin, and resveratrol: From dietary sources to human MicroRNA modulation. Molecules 25: 63, 2020.

7. Huang TT, Lin HC, Chen CC, Lu CC, Wei CF, Wu TS, Liu FG and Lai HC: Resveratrol induces apoptosis of human nasopharyngeal carcinoma cells via activation of multiple apoptotic pathways. J Cell Physiol 226: 720-728, 2011.

8. Lin HY, Sun M, Tang HY, Simone TM, Wu YH, Grandis JR, Cao HJ, Davis PJ and Davis FB: Resveratrol causes COX-2- and p53-dependent apoptosis in head and neck squamous cell cancer cells. J Cell Biochem 104: 2131-2142, 2008

9. Berta GN, Salamone P, Sprio AE, Di Scipio F, Marinos LM, Sapino S, Carlotti ME, Cavalli R and Di Carlo F: Chemoprevention of 7,12-dimethylbenz[a]anthracene (DMBA)-induced oral carcinogenesis in hamster cheek pouch by topical application of resveratrol complexed with 2-hydroxypropyl-beta-cyclodextrin Oral Oncol 46: 42-48, 2010.

10. Tyagi A, Gu M, Takahata T, Frederick B, Agarwal C, Siriwardana S, Agarwal R and Sclafani RA: Resveratrol selectively induces DNA Damage, independent of Smad4 expression, in its efficacy against human head and neck squamous cell carcinoma. Clin Cancer Res 17: 5402-5411, 2011.

11. Patel KR, Scott E, Brown VA, Gescher AJ, Steward WP and Brown K: Clinical trials of resveratrol. Ann N Y Acad Sci 1215: 161-169, 2011.

12. Brown VA, Patel KR, Viskaduraki M, Crowell JA, Perloff M, Booth TD, Vasilinin G, Sen A, Schinas AM, Piccirilli G, et al: Repeat dose study of the cancer chemopreventive agent resveratrol in healthy volunteers: Safety, pharmacokinetics, and effect on the insulin-like growth factor axis. Cancer Res 70: 9003-9011, 2010.
13. Patel KR, Brown VA, Jones DJ, Britton RG, Hemingway D, Miller AS, West KP, Booth TD, Perloff M, Crowell JA, et al: Clinical pharmacology of resveratrol and its metabolites in colorectal cancer patients. Cancer Res 70: 7392-7399, 2010.

14. Chow HH, Garland LL, Hsu CH, Vining DR, Chew WM, Miller JA, Perloff M, Crowell JA and Alberts DS: Resveratrol modulates drugand carcinogen-metabolizing enzymes in a healthy volunteer study. Cancer Prev Res (Phila) 3: 1168-1175, 2010.

15. la Porte C, Voduc N, Zhang G, Seguin I, Tardiff D, Singhal N and Cameron DW: Steady-State pharmacokinetics and tolerability of trans-resveratrol $2000 \mathrm{mg}$ twice daily with food, quercetin and alcohol (ethanol) in healthy human subjects. Clin Pharmacokinet 49: 449-454, 2010.

16. Almeida L, Vaz-da-Silva M, Falcao A, Soares E, Costa R, Loureiro AI, Fernandes-Lopes C, Rocha JF, Nunes T, Wright L and Soares-da-Silva P: Pharmacokinetic and safety profile of trans-resveratrol in a rising multiple-dose study in healthy volunteers. Mol Nutr Food Res 53 (Suppl 1): S7-S15, 2009.

17. Boocock DJ, Faust GE, Patel KR, Schinas AM, Brown VA, Ducharme MP, Booth TD, Crowell JA, Perloff M, Gescher AJ, et al: Phase I dose escalation pharmacokinetic study in healthy volunteers of resveratrol, a potential cancer chemopreventive agent, Cancer Epidemiol Biomarkers Prev 16: 1246-1252, 2007.

18. Juan ME, Gonzalez-Pons E and Planas JM: Multidrug resistance proteins restrain the intestinal absorption of trans-resveratrol in rats. J Nutr 140: 489-495, 2010.

19. Shirakami Y, Shimizu M and Moriwaki H: Cancer chemoprevention with green tea catechins: From bench to bed. Curr Drug Targets 13: 1842-1857, 2012.

20. Kim JW, Amin AR and Shin DM: Chemoprevention of head and neck cancer with green tea polyphenols. Cancer Prev Res (Phila) 3: 900-909, 2010.

21. Chakrawarti L, Agrawal R, Dang S, Gupta S and Gabrani R: Therapeutic effects of EGCG: A patent review. Expert Opin Ther Pat 26: 907-916, 2016.

22. Gan RY, Li HB, Sui ZQ and Corke H: Absorption, metabolism, anti-cancer effect and molecular targets of epigallocatechin gallate (EGCG): An updated review. Crit Rev Food Sci Nutr 58: 924-941, 2018.

23. Pisters KM, Newman RA, Coldman B, Shin DM, Khuri FR, Hong WK, Glisson BS and Lee JS: Phase I trial of oral green tea extract in adult patients with solid tumors. J Clin Oncol 19: 1830-1838, 2001.

24. Shanafelt TD, Call TG, Zent CS, LaPlant B, Bowen DA, Roos M, Secreto CR, Ghosh AK, Kabat BF, Lee MJ, et al: Phase I trial of daily oral Polyphenon $\mathrm{E}$ in patients with asymptomatic Rai stage 0 to II chronic lymphocytic leukemia. J Clin Oncol 27: 3808-3814, 2009.

25. Tsao AS,Liu D,Martin J,Tang XM,Lee JJ,El-Naggar AK, Wistuba I, Culotta KS, Mao L, Gillenwater A, et al: Papadimitrakopoulou V, Phase II randomized, placebo-controlled trial of green tea extract in patients with high-risk oral premalignant lesions. Cancer Prev Res (Phila) 2: 931-941, 2009

26. Basu A, Du M, Sanchez K, Leyva MJ, Betts NM, Blevins S, Wu M, Aston CE and Lyons TJ: Green tea minimally affects biomarkers of inflammation in obese subjects with metabolic syndrome. Nutrition 27: 206-213, 2011.

27. Kumar NB, Pow-Sang J,Egan KM, Spiess PE, Dickinson S, Salup R, Helal M, McLarty J, Williams CR, Schreiber F, et al: Randomized, placebo-controlled trial of green tea catechins for prostate cancer prevention. Cancer Prev Res (Phila) 8: 879-887, 2015.

28. Shin DM: Oral cancer prevention advances with a translational trial of green tea. Cancer Prev Res (Phila) 2: 919-921, 2009.

29. Amin AR, Wang D, Zhang H, Peng S, Shin HJ, Brandes JC, Tighiouart M, Khuri FR, Chen ZG and Shin DM: Enhanced anti-tumor activity by the combination of the natural compounds (-)-epigallocatechin-3-gallate and luteolin: Potential role of p53. J Biol Chem 285: 34557-34565, 2010.

30. Amin AR, Haque A, Rahman MA, Chen ZG, Khuri FR and Shin DM: Curcumin induces apoptosis of upper aerodigestive tract cancer cells by targeting multiple pathways. PLoS One 10: e0124218, 2015.

31. Reiss M,Pitman SW and Sartorelli AC: Modulation of the terminal differentiation of human squamous carcinoma cells in vitro by all-trans-retinoic acid. J Natl Cancer Inst 74: 1015-1023, 1985

32. Zhao M, Sano D, Pickering CR, Jasser SA, Henderson YC, Clayman GL, Sturgis EM, Ow TJ, Lotan R, Carey TE, et al: Assembly and initial characterization of a panel of 85 genomically validated cell lines from diverse head and neck tumor sites. Clin Cancer Res 17: 7248-7264, 2011. 
33. R Core Team (2013). R: A language and environment for statistical computing. R Foundation for Statistical Computing, Vienna, Austria. URL http://www.R-project.org/. 2020.

34. Shen LF, Zhao X, Zhou SH, Lu ZJ, Zhao K, Fan J and Zhou ML: In vivo evaluation of the effects of simultaneous inhibition of GLUT-1 and HIF-1 $\alpha$ by antisense oligodeoxynucleotides on the radiosensitivity of laryngeal carcinoma using micro ${ }^{18} \mathrm{~F}-\mathrm{FDG}$ PET/CT. Oncotarget 8: 34709-34726, 2017.

35. Zhu S, Moore TW, Lin X, Morii N, Mancini A, Howard RB, Culver D, Arrendale RF, Reddy P, Evers TJ, et al: Synthetic curcumin analog EF31 inhibits the growth of head and neck squamous cell carcinoma xenografts. Integr Biol (Camb) 4: 633-640, 2012.

36. Zhang X, Chen ZG, Choe MS, Lin Y, Sun SY, Wieand HS, Shin HJ, Chen A, Khuri FR and Shin DM: Tumor growth inhibition by simultaneously blocking epidermal growth factor receptor and cyclooxygenase- 2 in a xenograft model. Clin Cancer Res 11: 6261-6269, 2005.

37. Cancer Genome Atlas Network: Comprehensive genomic characterization of head and neck squamous cell carcinomas. Nature 517: 576-582, 2015.

38. Leemans CR, Snijders PJF and Brakenhoff RH: The molecular landscape of head and neck cancer. Nat Rev Cancer 18: 269-282, 2018.

39. Anisuzzaman AS, Haque A, Rahman MA, Wang D, Fuchs JR Hurwitz S, Liu Y, Sica G, Khuri FR, Chen ZG, et al: Preclinical in vitro, in vivo, and pharmacokinetic evaluations of FLLL12 for the prevention and treatment of head and neck cancers. Cancer Prev Res (Phila) 9: 63-73, 2016.

40. Gotwals P, Cameron S, Cipolletta D, Cremasco V, Crystal A, Hewes B, Mueller B, Quaratino S, Sabatos-Peyton C, Petruzzelli L, et al: Prospects for combining targeted and conventional cancer therapy with immunotherapy. Nat Rev Cancer 17: 286-301, 2017.

41. Carneiro BA and El-Deiry WS: Targeting apoptosis in cancer therapy. Nat Rev Clin Oncol 17: 395-417, 2020.

42. Faber AC, Ebi H, Costa C and Engelman JA: Apoptosis in targeted therapy response: The role of BIM. Adv Pharmacol 65: 519-542, 2012

43. Camidge DR, Pao W and Sequist LV: Acquired resistance to TKIs in solid tumors: Learning from lung cancer. Nat Rev Clin Oncol 11: 473-481, 2014.

44. Hata AN, Engelman JA and Faber AC: The BCL2 family: Key mediators of the apoptotic response to targeted anticancer therapeutics. Cancer Discov 5: 475-487, 2015.

45. Agrawal N, Frederick MJ, Pickering CR, Bettegowda C, Chang K, Li RJ,Fakhry C, Xie TX, Zhang J, Wang J, et al: Exome sequencing of head and neck squamous cell carcinoma reveals inactivating mutations in NOTCH1. Science 333: 1154-1157, 2011

46. Stransky N, Egloff AM, Tward AD, Kostic AD, Cibulskis K, Sivachenko A, Kryukov GV, Lawrence MS, Sougnez C, McKenna A, et al: The mutational landscape of head and neck squamous cell carcinoma. Science 333: 1157-1160, 2011.
47. Poetsch M, Lorenz G and Kleist B: Detection of new PTEN/MMAC1 mutations in head and neck squamous cell carcinomas with loss of chromosome 10. Cancer Genet Cytogenet 132: 20-24, 2002

48. Shao X, Tandon R, Samara G, Kanki H, Yano H, Close LG, Parsons R and Sato T: Mutational analysis of the PTEN gene in head and neck squamous cell carcinoma. Int J Cancer 77: 684-688, 1998

49. Madera D, Vitale-Cross L, Martin D, Schneider A, Molinolo AA, Gangane N, Carey TE, McHugh JB, Komarck CM, Walline HM, et al: Prevention of tumor growth driven by PIK3CA and HPV oncogenes by targeting mTOR signaling with metformin in oral squamous carcinomas expressing OCT3. Cancer Prev Res (Phila) 8: 197-207, 2015.

50. Sun ZJ, Zhang L, Hall B, Bian Y, Gutkind JS and Kulkarni AB Chemopreventive and chemotherapeutic actions of mTOR inhibitor in genetically defined head and neck squamous cell carcinoma mouse model. Clin Cancer Res 18: 5304-5313, 2012.

51. Vitale-Cross L, Molinolo AA, Martin D, Younis RH, Maruyama T, Patel V, Chen W, Schneider A and Gutkind JS: Metformin prevents the development of oral squamous cell carcinomas from carcinogen-induced premalignant lesions. Cancer Prev Res (Phila) 5: 562-573, 2012.

52. Shin DM, Zhang H, Saba NF, Chen AY, Nannapaneni S, A min AR, Muller S, Lewis M, Sica G, Kono S, et al: Chemoprevention of head and neck cancer by simultaneous blocking of epidermal growth factor receptor and cyclooxygenase-2 signaling pathways: Preclinical and clinical studies. Clin Cancer Res 19: 1244-1256, 2013.

53. Bartel DP: MicroRNAs: Target recognition and regulatory functions. Cell 136: 215-233, 2009.

54. Hammond SM: An overview of microRNAs. Adv Drug Deliv Rev 87: 3-14, 2015

55. Zan L, Chen Q, Zhang L and Li X: Epigallocatechin gallate (EGCG) suppresses growth and tumorigenicity in breast cancer cells by downregulation of miR-25. Bioengineered 10: 374-382, 2019.

56. Zhu Y, Huang Y, Liu M, Yan Q, Zhao W, Yang P, Gao Q, Wei J, Zhao W and Ma L: Epigallocatechin gallate inhibits cell growth and regulates miRNA expression in cervical carcinoma cell lines infected with different high-risk human papillomavirus subtypes. Exp Ther Med 17: 1742-1748, 2019.

57. Fu J, Shrivastava A, Shrivastava SK, Srivastava RK and Shankar S: Triacetyl resveratrol upregulates miRNA200 and suppresses the Shh pathway in pancreatic cancer: A potential therapeutic agent. Int J Oncol 54: 1306-1316, 2019.

This work is licensed under a Creative Commons Attribution-NonCommercial-NoDerivatives 4.0 International (CC BY-NC-ND 4.0) License. 